DOI: $10.5216 /$ racs.v4.59298

\title{
Mi experiencia en el Método Inductivo Intercultural
}

\author{
Alfredo Martínez López ${ }^{1}$
}

\section{RESUMEN}

El texto presenta mi experiencia con el Método Inductivo Interculturall en las escuelas del estado de Oaxaca / México. Mi interés en apropiarse de esta metodología, expresada en la elaboración del calendario socionatural y en las tarjetas de aprendizaje, deriva de mis raíces indígenas, pues como muchos vivía la necesidad de afirmarla, valorizando mi propia cultura.

PALABRAS CLAVE: Método inductivo intercultural. Calendario socionatural. Indio.

\section{Minha experiência no Método Indutivo Intercultural}

\section{RESUMO}

O texto apresenta minha experiência com o Método Indutivo Intercultural em escolas do estado de Oaxaca/México. Meu interesse em se apropriar desta metodologia, expressa na elaboração do calendário socionatural e nos cartões de aprendizagem, decorre de minhas raízes indígenas, pois como muitos viviam a necessidade de afirmá-la, valorizando minha própria cultura zapoteca.

PALAVRAS-CHAVE: Método Indutivo Intercultural. Calendário socionatural. Indígena.

Tiene varios años ya, en el 2008, cuando estaba cursando el octavo semestre de la Licenciatura de Educación Primaria y Preescolar en la Universidad Pedagógica Nacional, se publicó la convocatoria para cursar unos diplomados. Sin pedir detalle alguno me inscribí al proceso de selección y después de unos días al checar la lista de aceptados no aparecía mi nombre; no hice caso alguno simplemente lo deje pasar, pero ya transcurrido las primeras sesiones de este, algunos alumnos inscritos ya no asistían, por lo que la coordinadora realizo nuevamente la invitación para que otros interesados se integraran, así fue como me incorpore

\footnotetext{
${ }^{1}$ Profesor de Educación Indígena. Povo Zapoteca. Oaxaca, México. E-mail: articulandoeconstruindosaberes@gmail.com.
} 
a los diplomados de "Sistematización del conocimiento indígena y diseños de materiales interculturales y bilingües” en el cual conocí el Método Inductivo Intercultural.

Mi interés en apropiarme de la metodología y de llevarlo a la práctica se deriva de mis raíces indígenas, ya que como muchos, vivía interiorizado en la ambivalencia y en la negación de mi cultura propia, además el afianzar una estima hacia los compañeros y compañeras que seguíamos este mismo trabajo en diversos lugares hizo que no me alejara de ellos y me motivó a seguir poniendo en práctica lo ya aprendido.

Al termino de los diplomados tenía la posibilidad de titularme con la metodología presentando mi propuesta pedagógica al que le puse el título "Las actividades sociales y productivas como fuente de conocimiento en educación intercultural”, la cual fue aprobada por unanimidad por los sinodales; en ella puse en práctica lo aprendido durante los diplomados realizando una investigación en la comunidad donde trabajaba teniendo como producto un calendario socionatural y dos tarjetas de autoaprendizaje, una titulada "cultivemos nuestra grana cochinilla fina" y la otra "cosechemos nuestro durazno". Durante el desarrollo de esta experiencia tuve muchas satisfacciones que fueron: el acercarme más a la comunidad donde trabajo, el ganarme la confianza de los padres de familia al escucharlos e interactuar con ellos en sus actividades cotidianas, el sentirme parte de la comunidad y menos ajeno a ella, con mis alumnos la experiencia fue satisfactoria y motivó a darle valor a las actividades que se realizan en la comunidad.

Actualmente como docente de educación indígena he laborado en diversas comunidades, en distintas regiones de mi estado. En todas he puesto en práctica el MII, pero no de la misma manera, esto depende de las características de la comunidad, en aquellas que son comunidades indígenas o comunidades originarias, como ahora está en auge llamarlas, el método revitaliza la cultura al trabajar actividades relacionadas con la gastronomía, la fiesta, la agricultura, los rituales, los juegos tradicionales como el papalote, las canicas, el trompo, etc.

Debido a que me he cambiado varias veces de comunidades, y con la intención de conjuntar los diversos procesos y materiales que el MII propone, he realizado tarjetas de autointeraprendizaje utilizando fotografías; esto agiliza la realización del material ya que el dibujo resulta laborioso hacerlo y requiere de más tiempo. 
Figura 1 - Tarjeta de auto-interaprendizaje con fotografías.

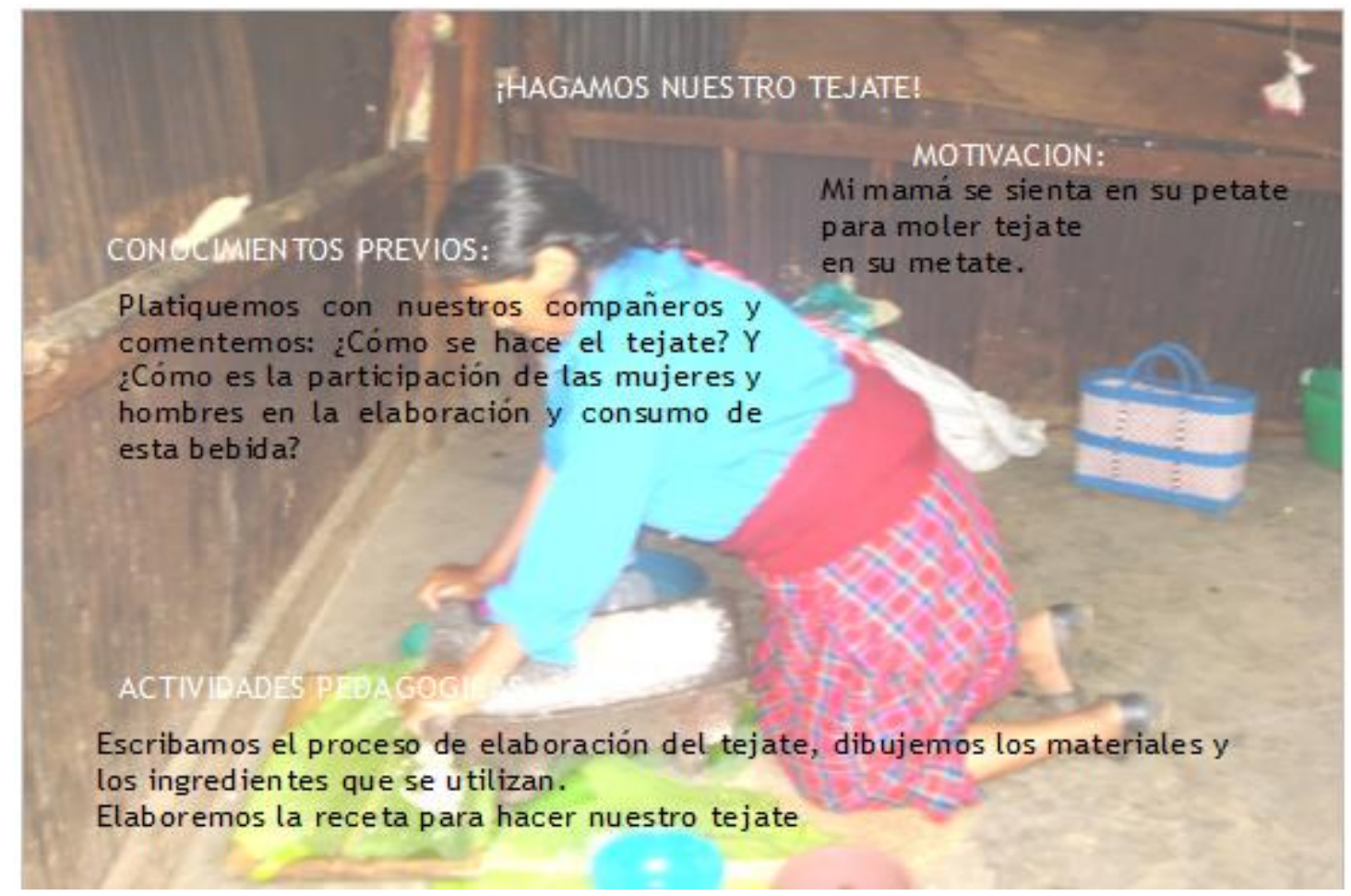

Fonte: Alfredo Martínez López.

Figura 1a - Tarjeta de auto-interaprendizaje con fotografías.

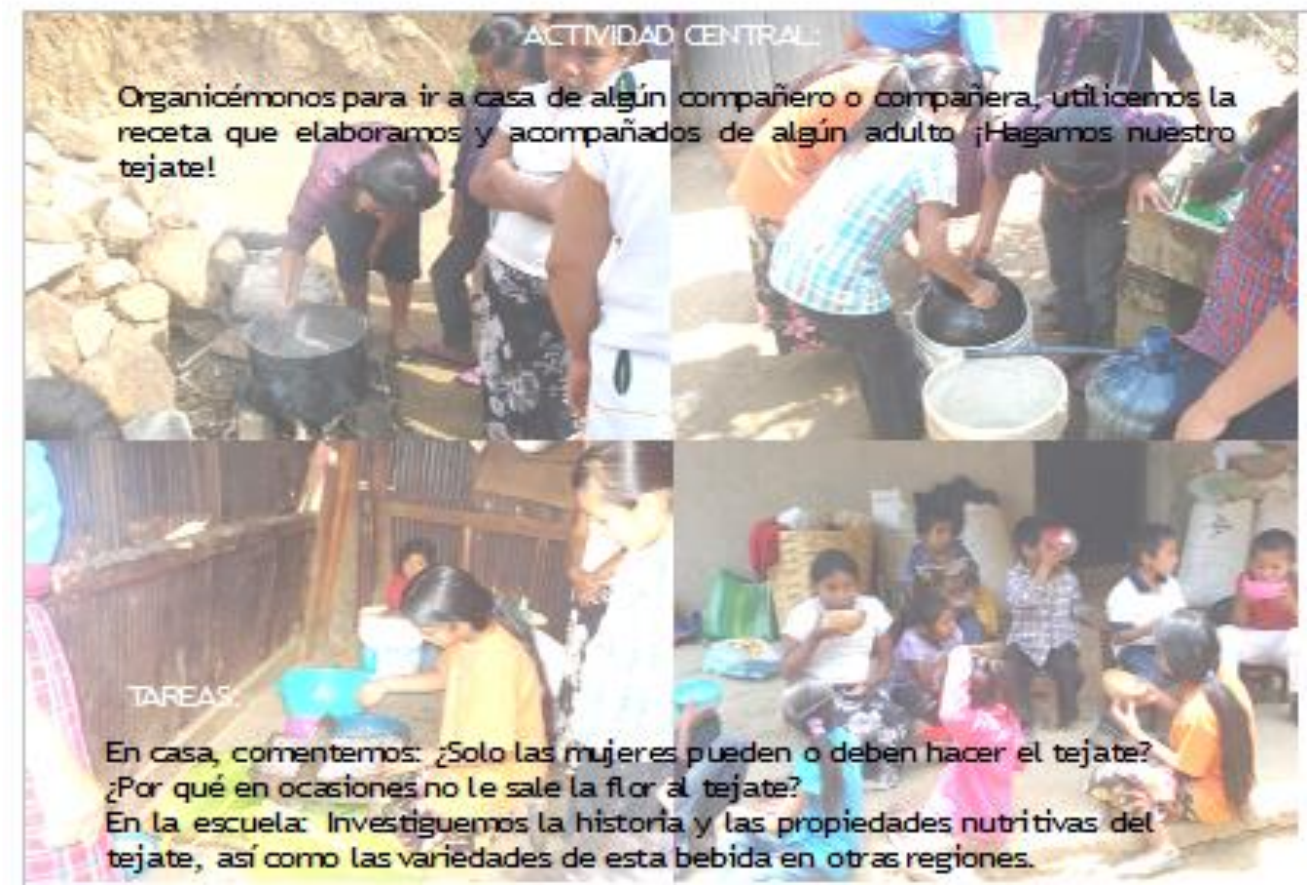

Fonte: Alfredo Martínez López. 
En contextos más cercanos a la cuidad, en donde he tenido también experiencia, la dinámica cambia, ya que me he encontrado con otros factores que hacen tomar otra dinámica de trabajo para adecuar el MII. Como ejemplo: en la escuela donde actualmente laboro es una comunidad que se ha venido formando hace apenas 3 décadas, con familias provenientes de diferentes regiones del estado, inclusive familias que vienen de otros estados del país, los motivos de su migración son distintos; algunos por voluntad propia por tener ese "deseo de superar a su familia" como ellos lo dicen, otros más por conflictos o problemas fueron expulsados de sus comunidades de origen, otros por estar afiliados a organizaciones sociales se han hecho "ganador" de un lote, situación que los obliga a venirse a vivir aquí para cuidarlo. Todas estas familias tienen historias de vidas diferentes, lo que hace que no tengan una identidad arraigada en la nueva cultura que están creando ni en el nuevo territorio donde habitan. En consecuencia los niños y niñas que atiendo también tienen estos problemas, no se identifican con la cultura de los pueblos de origen de sus padres ni han logrado afianzar una identidad en la colonia en que viven. Esto para la implementación del MII ha sido un reto, lo que he realizado es buscar un punto de cohesión para todos, me he centrado en realizar actividades con el huerto escolar como un primer acercamiento de los padres de familia con actividades fuera del currículo nacional.

Figura 2 - Actividades relacionadas con el huerto escolar.
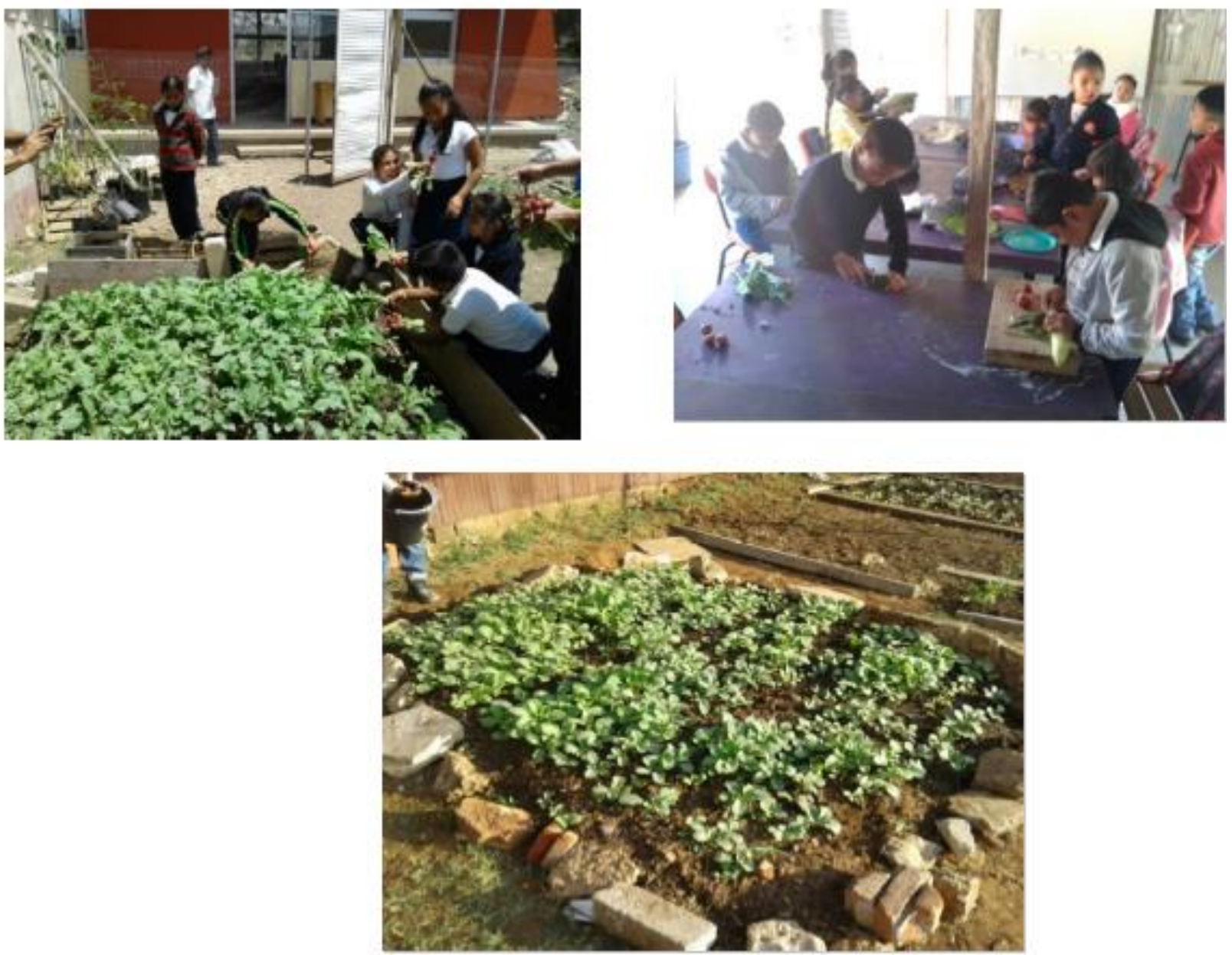

Fonte: Alfredo Martínez López. 
En ocasiones he realizado actividades relacionadas con el mejoramiento escolar, por ejemplo: ante la necesidad de contar con sombra para el patio escolar, realizamos una tarjeta de auto-interaprendizaje que consistió en plantar árboles en el patio de la escuela, varios de estos árboles siguen vivos y van creciendo para en unos años tener sombra con esto valorando la importancia y el cuidado del medio ambiente.

Figura 3 - Tarjeta de auto-interaprendizaje de una actividad en mejoramiento de la escuela.

\section{¡Vamos al patio de la escuela a plantarnuestros árboles para que nos} den oxigeno y jugar bajo su sombra!

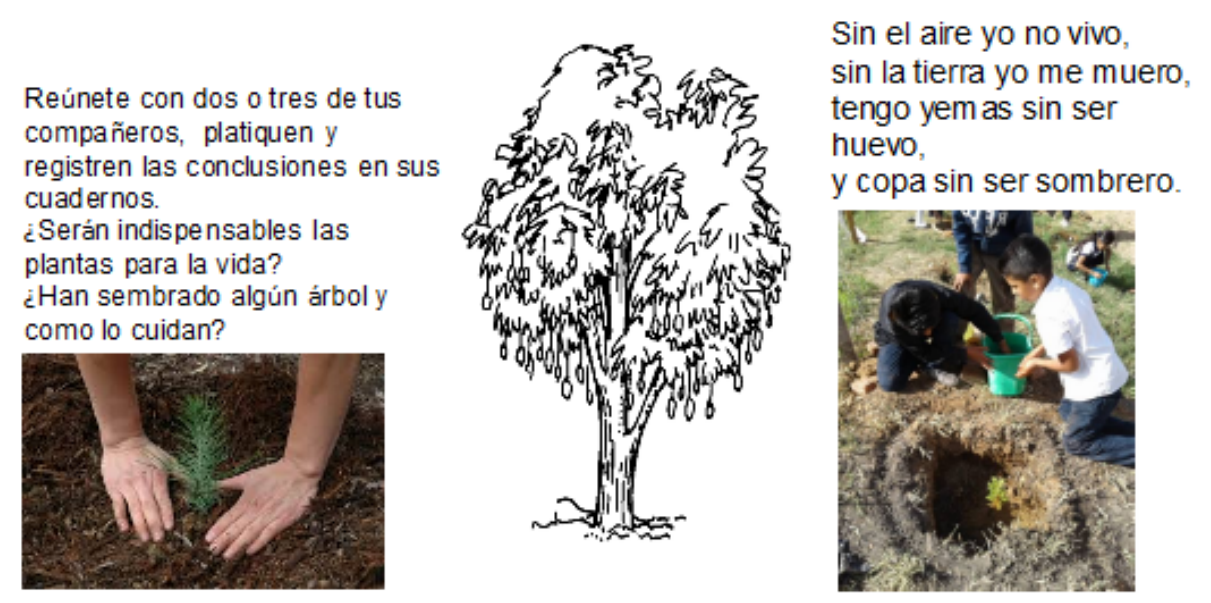

Dibuja y describe en tu cuaderno los tipos de arboles que cresen en tu colonia

Fonte: Alfredo Martínez López.

En otro contexto el estado de Oaxaca, es caracterizado por estar inmerso en una constante lucha magisterial, escenario donde también las instancias del sindicato abanderan una educación alternativa teniendo varias propuestas para esto. Por tanto; en particular he tenido la iniciativa de incorporar los aportes del MII en las propuestas de educación alternativa que propone el magisterio; debido a que son propuestas hermanadas y además se articulan en diversos puntos. 
Figura 4 - Tarjeta de auto-interaprendizaje de una actividad en mejoramiento de la escuela.

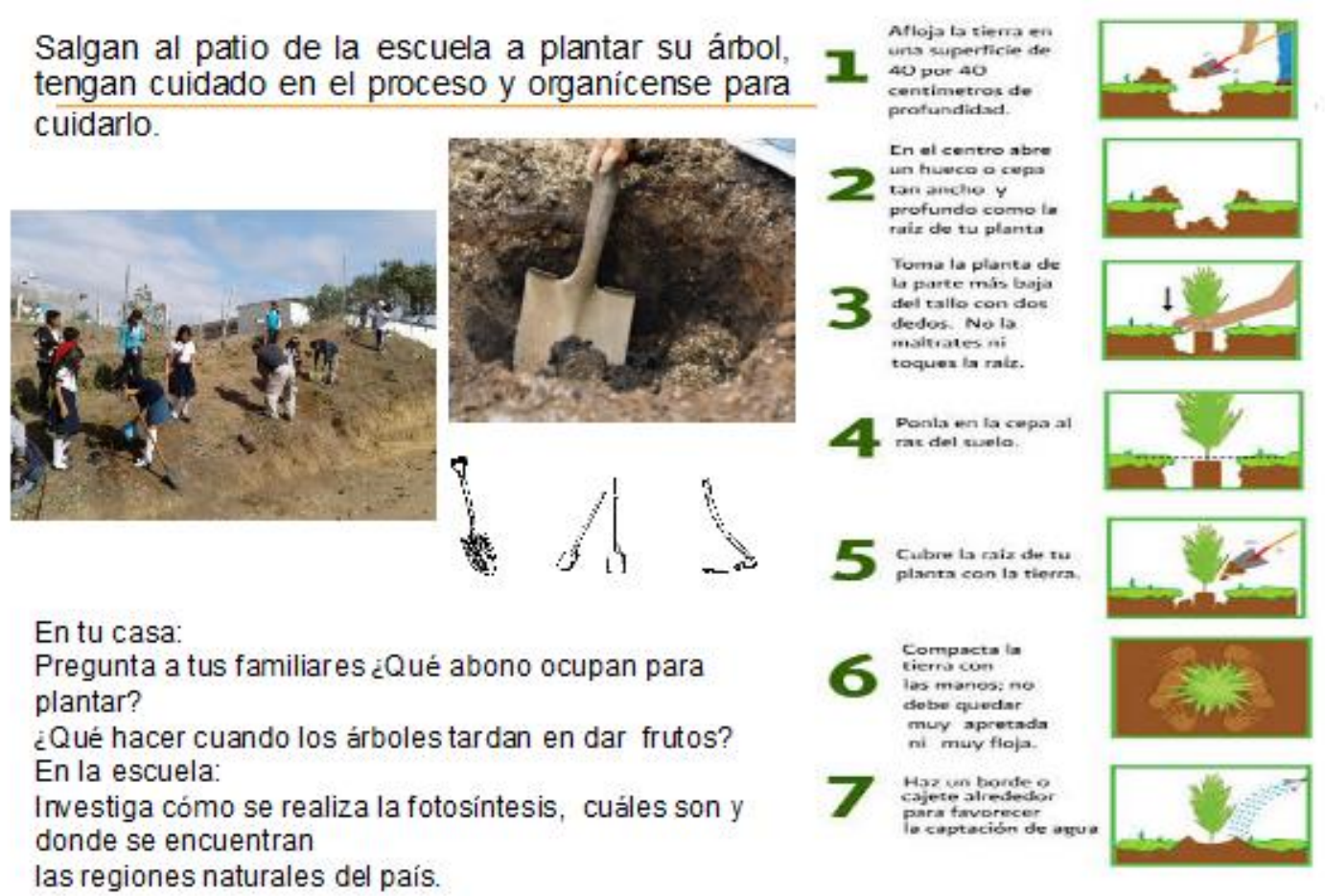

Fonte: Alfredo Martínez López.

Por una u otra manea he tenido mucha motivación para después de 10 años de haber conocido y empezado a trabajar el MII, siga creyendo en el método. Concluyo con la seguridad de que para seguir dándole vida debo de concatenar los diferentes aportes que actualmente caminen por el mismo sendero, con propuestas hermanadas; de lo contrario en algún momento se disiparía el espíritu de quienes han hecho posible esta propuesta.

\section{Referência}

BERTELY, Maria Busquets. SARTORELLO, Stefano. Milpas Educativas: Laboratórios socionaturales vivos para el Buen Vivir. Universidad Iberoamericana, 2017.

Submetido em 02 de julho de 2019.

Aceito em 05 de agosto de 2019.

Publicado em 08 de agosto de 2019. 